Deutangen geometrische Operationen suchte, die den Rechnungsoperationen parallel gehen. Dabei spielt $\Omega$ in gewisser Beziehungdie Rolle einer imaginären Einheit, jedoch ist $Q^{2}=0$; man sieht jetzt auch, dass ein Octonion $Q$ von 8 Scalargrößen abhängt, woraus sich der Name erklärt. Es werden, analog wie in der Quaternionentheorie, die symbole $K Q, S Q, V Q, T Q, U Q_{E}$ definiert, auch die Begriffe der linearen Vectorfunction und die Anwendung des Operationszeichens $\nabla$ verallgemeinert. Die weitern Rechnungsregeln fallen ziemlich analng mit denen der Quaternionenlehre aus, nur entsprechend complicierter. Ein Motor kann dabei in der Form $\omega+\Omega \sigma$ dargestellt werden, wobei $\omega$ und $\sigma$ Rotoren sind (im wesentlichen identisch mit den Linientheilen Grassmanns, d. h. von den Vectoren dadurch unterschieden, dass sie nicht nur der Richtung sondern auch der Lage nach bestimmt sind; analog unterscheidet der Verfasser neben den gewöhnlichen Quaternionen noch die "Axiale", deren Drehungsachse auch der Lage nach bestimmt ist). Auch die Ideen und Methoden von Grassmanns Ausdehnungslehre (1862) werden in ausgedehntem Maße herangezogen. Z. B. wird die Frage eingehend erörtert, inwieweit die "reciproken" Schrauben Balls als "normal" im Sinne Grassmanns aufgefasst werden können. Die Theorie wird hauptsächlich auf die Lehre von der Bewegung eines starren Körpers and überhaupt auf die Probleme angewendet, welche den Gegenstand von Balls "Theory of the screws" ausmachen.

Das vorliegende Werk dürfte den allgemeinsten und abstractesten Calcul mit räumlichen Größen and räumlichen Operationen darstellen, den wir bisher besitzen. Dementsprechend darf man sich auch das Studium des Buches nicht leicht vorstellen, wie schon daraus entnommen werden mag, dass sowohl die volle Beherrschung der Quaternionenlehre, als auch die Kenntnis der Ausdehnungslehre vorausgesetzt wird. Aber es ist zweifellos, dass man mit diesem Calcul einen noch größeren Kreis von Problemen beherrscht als mit der Quaternionenlehre. Dex Verfasser wirkt an der Universität Tasmania (Australien) and ist durch sein Werk "Utility of Quaternions in Physics" (London, Macmillan, 1893) in der wissenschaftlichen Welt schon bekannt.

\title{
Konrad Zindler.
}

Heron von Alexandria. Von W. Schmidt. (Sonderabdruck aus den ,Neven Jahrbuichern für das classische Alterthum, Geschichte und deutsche Literatur") Mit 39 Abbildungen auf 3 Tafeln. 15 S. Lex. $8^{0}$. B. G. Teubner, Leipzig, 1899. Ladenpreis 0.80 M.

Der vor Kurzem ausgegebene erste Band der in der Bibliotheca Teubneriana erschienenen neuen Ausgabe von Heron's Werken, welcher von dem Verfasser der vorliegenden Schrift besorgt wurde, enthält neben einer Einleitung in welcher u. a. die sog. Heronische Frage eingehend erörtert wird, die „Druckwerke," und die "Automatentheater" nebst einigen Ergänzungen aus Philo von Byzanz und aus Vitruv, griechisch und deutsch nebst Figuren, welche auf Grund der handschriftlichen Figuren reconstruiert sind. Der Inhalt dieser zwei Heronischen Werke wird uns nebst den nöthigen Erläuterungen in der oben genannten Broschüre in klarer, äuferst ansprechender Weise vorgeführt.

Mathematische Aufgaben. Von Dr. Willy Timpe, Oberlehrer. (Wissenschaftliche Beilage zum Jahresbericht der sechsten 